\title{
The Changing Picture of Pseudotumor Cerebri in Children
}

\author{
R. Couch, P.R. Camfield, J.A.R. Tibbles
}

\begin{abstract}
Among 38 children with pseudotumor cerebri only 3 cases were due to ear disease or its complications. The commonest cause was a refeeding syndrome either due to nutritional deprivation or as an early finding in the treatment of cystic fibrosis. It is uncommon to find the cause of pseudotumor in older children but in those under 6 years the cause was found in $85 \%$. Because of the adverse effects of steroids we use this treatment in the more resistant cases.

RÉSUMÉ: Modification du tableau de la "pseudotumor cerebri" chez l'enfant Seulement 3 cas étaient dus à des maladies de l'oreille ou leurs complications sur un total de 38 enfants souffrant de pseudotumor cerebri. La cause la plus fréquente semble être un syndrome de renutrition, soit à la suite d'une déprivation nutritionnelle, soit au début du traitement d'une fibrose kystique. S'il est difficile d'établir la cause d'une "pseudotumor" chez l'enfant de plus de 6 ans, sous cet âge la cause peut être déterminée chez $85 \%$ des patients. A cause des effets secondaires des stéroïdes, nous n'employons ce traitement que chez les cas les plus résistants.
\end{abstract}

Can. J. Neurol. Sci. $1985 ; 12: 48-50$

Pseudotumor cerebri is a syndrome characterized by symptoms and signs of increased intracranial pressure without evidence of a mass lesion or hydrocephalus. The condition differs from other causes of increased intracranial presssure in that the level of consciousness is not altered. The diagnosis is one of exclusion after documentation of raised intracranial pressure with normal cerebrospinal fluid and CT scan or ventriculogram where appropriate. The syndrome was first described by Quincke in 1897 (H. Quincke, 1897). Numerous studies have since been reported, but the majority involve adult patients (Boddie, Hanna and Bradley, 1974; Johnston and Patterson, 1974; Weisberg, 1975). In these reports obese females predominate. The few papers devoted exclusively to pseudotumor cerebri in children have identified the major cause as otitis media, with or without venous sinus obstruction (Greer, 1967; Grant, 1971) or other infections (Rose and Matson, 1967). We have reviewed the cases of pseudotumor cerebri at the Izaak Walton Killam Hospital for Children over the past 13 years and will show that otitis media is no longer the major precipitating event. In addition, we have reviewed our treatment of these patients, and based on our own experience outline our recommendations for the management of pseudotumor cerebri in children.

\section{MethoDS}

The charts of all patients discharged with a diagnosis of pseudotumor cerebri from 1968 to 1981 were reviewed. All the patients had been seen by at least one of the authors. For the diagnosis to be accepted the following criteria were met: (1) clinical evidence of increased intracranial pressure, (2) confirmation of raised intracranial pressure by lumbar puncture, split sutures on skull $x$-ray or bulging anterior fontanelle, and in most cases, (3) a CT scan or ventriculogram that excluded a mass lesion or hydrocephalus. Eight patients were accepted into the study who did not have a CT scan or ventriculogram. These patients were all less than 2 years old and met the other 2 criteria for the diagnosis of pseudotumor cerebri. In total there were 38 patients, 21 of them presenting since 1978.

\section{RESULTS}

Thirty-eight children with a diagnosis of pseudotumor cerebri have been seen since 1968. They have ranged in age from 3 months to 14 years, with $50 \%$ less than 6 years old. The sex ratio has been approximately equal (17 males, 20 females).

Presenting symptoms have been those expected with increased intracranial pressure: headache in 22 , nausea and vomiting in 17 , and visual disturbances in 15 . A few patients had unusual complaints such as neck pain (4), unsteady gait (3), intermittent paresthesia (2), and one patient complained of a "swishing" noise in both ears which correlated with an audible generalized cranial bruit.

Bilateral papilledema was present in 26 patients: of the 12 who were without papilledema, 7 were less than 1 year of age

Presented in part, at the Canadian Congress of Neurological Sciences Meeting, June 1982, Toronto.

From the Neurology Service. Izaak Walton Killam Hospital for Children and Dalhousie University. Halifax, Nova Scotia.

Received May 22, 1984. Accepted in revised form October 15, 1984.

Reprint requests to: Dr. P. Camfield, Department of Paediatric Neurology, Izaak Walton Killam Hospital for Children, 5850 University Ave., P.O. Box 3070. Halifax, Nova Scotia. Canada B3J 3G9 
and had a bulging anterior fontanelle, and the other 5 had split sutures on roentgenograms of the skull. Visual acuity was altered in only 4 of 24 patients tested, but physiologic blind spots were enlarged in 8 of 12 assessed. Four of these 8 patients also had decreased visual acuity.

Cranial nerve palsies were present in 6 patients. Five had abducens nerve palsies and in 2 it was bilateral. One of these patients also had a facial nerve weakness and a separate child had an isolated trochlear nerve palsy. The signs all disappeared with resolution of the increased intracranial pressure.

The causes of pseudotumor cerebri in our patients are shown in Table 1. Only 3 children had acute otitis media, and in 2 of these, sinus thrombosis was demonstrated on arteriography. Two other cases were related temporally to a presumed viral infection. Head trauma, severe in one child, accounted for 2 further cases. Five patients were admitted for failure to thrive and were found to have pseudotumor cerebri on admission. A sixth undernourished patient developed the disorder on refeeding. A similar, but distinct, phenomenon was seen in 4 patients aged 4 - 7 months with cystic fibrosis (CF). Within 1 to 2 days of starting treatment for their C.F., these patients all developed raised intracranial pressure which resolved spontaneously in one or two weeks with no specific treatment. Other causes included the use of topical steroids, iron-deficiency anemia and radiation vasculitis in a 9 year old girl with acute lymphoblastic leukemia. There were 17 patients for whom no cause could be found. There were no cases related to obesity.

Table 1: Presumed Causes

\begin{tabular}{lc}
\hline \hline Documented Sinus Thrombosis & 2 \\
Otitis Media & 1 \\
Viral Infection & 2 \\
Head Trauma & 2 \\
Topical Steroids & 2 \\
Psychosocial and Nutritional Deprivation & 6 \\
Cystic Fibrosis & 4 \\
Iron Deficiency & 1 \\
Radiation Vasculitis & 1 \\
Unknown & 17 \\
\hline
\end{tabular}

When compared by age (Table 2), it becomes evident that a cause is more likely to be found in the younger patient. Sixteen of 19 patients less than 6 years old had an etiologic factor identified, whereas none of nineteen over 11 years had a cause identified.

Table 2: Relation of Age to Detectable Cause

\begin{tabular}{ccc}
\hline \hline Age & Total Patients & Cause Found \\
\hline 6 years & 19 & 16 \\
$6-11$ years & 9 & 5 \\
$11-14$ years & 10 & 0 \\
\hline
\end{tabular}

Treatment varied as shown in Table 3 . Of the 13 patients who received only lumbar puncture, all had resolution of their symptoms and increased pressure with the L.P. Of those who received steroids, all but 2 had normal CSF pressure within 1 month of starting treatment. However, at least 6 became frankly cushingoid and 1 developed a reversible steriod-induced myopathy. Three patients required lumbo-peritoneal shunts. One was shunted immediately after presentation because of severely decreased visual acuity. The other 2 children received steroids initially, but in one vision continued to deteriorate despite steroids and the other failed to respond and became strikingly cushingoid.

Table 3: Treatment of Pseudotumor

\begin{tabular}{ll}
\hline \hline & \\
None & 7 \\
L.P. only & 13 \\
Steroid & 7 \\
Diuretic & 1 \\
Steroid \& Diuretic & 6 \\
Iron & 1 \\
Lumboperitoneal Shunt & 3 \\
\hline
\end{tabular}

Twenty-three patients were in remission within 1 month of starting treatment. Only 2 had evidence of increased pressure for longer than 1 year and both of these were deprived children who continue to do poorly in their home environment. Two patients have had a recurrence which responded to further treatment. Permanent visual impairment was seen in 2 patients both of whom had lumbo-peritoneal shunts. Both of them had presented late in the course of their illness and had impaired vision at that time. One is left with an acuity of $20 / 400$ in the right eye and $20 / 100$ in the left; the other is blind in one eye with an acuity of $20 / 50$ in the other eye.

\section{Discussion}

Previous reports of pseudotumor cerebri in children have identified otitis media as the commonest cause (Greer, 1967; Grant, 1971). In our series only 3 cases $(8 \%)$ could be attributed to ear disease and its complications. This change is probably related to the extensive and early use of antibiotics for middle ear effusion.

The commonest cause in our series, accounting for $26 \%$ of the cases, is a syndrome associated with malnutrition. The first type is the nutritional deprivation syndrome. Four of the six patients have been reported previously (Tibbles et al., 1972). These children typically are free of symptoms of raised pressure and may have separated sutures on skull $x$-ray when first seen, or have it develop during the first 2 months of refeeding (Capitanio and Kirkpatrick, 1969; Sonheimer, Grossman and Winchester, 1970 and Delevie and Nogrady, 1970). There is accelerated growth of the head size when they receive suitable nutrition, which suggests accelerated brain growth. In pigs fed protein calorie deficient diets, that bone growth is severely affected and this may account for the initial rise in intracranial pressure with involvement of the skull vault. Refeeding presumably permits more rapid growth of the brain than the skull vault which would explain the development of raised intracranial pressure that occurs with refeeding. 
The second type of pseudotumor syndrome linked with malnutrition occurs in children with cystic fibrosis. In our 4 patients, the onset of raised intracranial pressure occurred within 3 days of starting treatment for their C.F. This is earlier than in most other patients with C.F. including 2 infants reported by Keating and Feigin in 1973 who had pseudotumor with evidence of vitamin A deficiency that appeared about 2 months after the onset of treatment. The rapid early onset and rapid resolution in our patients together with 3 others in the literature (Bray and Herbst, 1973; Roach and Sinal, 1980) suggests that some mechanism other than differential brain growth is occurring. This is supported by normal vitamin $A$ levels in 2 of our patients with CF cystics prior to treatment, and also in 2 of them a peculiar picture of irritability and profound misery without any fever for the first few days of their raised intracranial pressure. Possible explanations include transient toxic effects of treatment or possible shifts in fluid compartments of the brain. Since the finding is transient it is appropriate to recognize the natural history of the condition at its time of occurrence and to await its spontaneous resolution in those cases that occur early.

An identifiable cause in the child older than 11 years is uncommon in our experience. We found none of these patients to be obese and there was no evidence of lead poisoning, hyper-or hypovitaminosis A, or tetracycline use. None had a perimenarchal syndrome as postulated by Greer (Greer, 1964).

Two (5\%) of the 38 patients had severe visual sequelae. This incidence is similar to other series (Johnston and Patterson, 1974; Rush, 1980). Both our patients had marked visual impairment at presentation. However, Rush has shown that the visual outcome is not related to the duration of symptoms, the degree of papilledema, the presence of obscurations or the incidence of recurrent increased intracranial pressure (Rush, 1980).

Although facial nerve weakness is uncommon in association with pseudotumor, in 3 cases in children reported by Chutorian et al. (1977) the authors speculated on an underlying common infectious etiology to both conditions. In our patient, there was a recurrence of the pseudotumor and also a recurrence of the facial palsy at the same time, but on the opposite side. Less common is trochlear palsy but this too has been reported before (Halpern and Gordon, 1981).

Clearly the reasons for treatment are relief of symptoms and preservation of vision. The initial L.P. resulted in remission in about one-third (13 of 38). Another 7 patients received no treatment and also had rapid spontaneous remissions. In Weisberg's series 55 of 105 patients had one or serial lumbar punctures which resulted in resolution of the raised pressure (Weisberg, 1975). The L.P. associated remission rate, plus the lack of any controlled therapeutic trials makes it difficult to assess treatment protocols. It seems clear from our patients that steroids, although effective in reducing the intracranial hypertension, have significant morbidity including weight gain, acne and steroid myopathy. A few series have suggested good results using diuretics in adults (Jefferson and Clark, 1976; Lubow and Kuhr, 1977).

On the basis of our experience we now recommend the following treatment protocol. After a normal CT scan has been obtained, a lumbar puncture should be performed and the pressure carefully measured. Enough fluid should be removed to lower the pressure towards normal. The visual acuity and fields should be documented as early as possible and followed serially. A second L.P. should be performed after 48 hours to identify the patients in whom raised pressure persists. In those with persistent elevated pressure a diuretic such as chlorthiazide, acetazolamide, or chlorthalidone should be administered in convential doses and a further L.P. performed after about 5 days. If the pressure remains increased, then a course of steroids for 4-8 weeks should be added. If the pressure does not respond to medical management or if there is evidence of deterioration of vision a lumboperitoneal shunt may be required. Children who have a recurrence may be treated as new cases, depending on clinical judgment.

In conclusion, we have outlined the changing pattern of pseudotumor cerebri in children and have demonstrated that it is uncommon to discern the cause in older children. Our experience of adverse effects of steroids leads us to reserve that treatment to the most resistant cases.

\section{REFERENCES}

Bray PF, Herbst J (1973) Pseudotumor cerebri as a sign of "catch-up" growth in cystic fibrosis. Am. J. Dis. Child. 126: 78-79.

Boddie HG, Banna M, Bradley WG (1974) “Benign" intracranial hypertension. Brain 97: 313-326.

Capitanio MT, Kirpatrick JA (1969) Widening of cranial sutures. Radiology 92: 53-59.

Chutorian AM, Gold AP, Brown CW (1977) Benign intracranial hypertension and Bell's palsy. N. Eng. J. Med. 296: 1214-1215.

DeLevie M, Nogrady MB (1970) Rapid brain growth upon restoration of adequate nutrition causing false radiologic evidence of increased intracranial pressure. J. Pediatr. 76: 523-528.

Grant DN (1971) Benign intracranial hypertension. A review of 79 cases in infancy and childhood. Arch. Dis. Child. 46: 651-655.

Greer M (1967) Benign intracranial hypertension. Ped. Clin. N. Am. 14: 819-830.

Greer M (1964) Benign intracranial hypertension. IV. Menarche, Neurology 14: 569-573.

Halpern JI, Gordon WH (1981) Trochlear Nerve Palsy as a false localizing sign. Ann. Ophthalmol. 9: 53-56.

Jefferson A, Clark J (1976) Treatment of benign intracranial hypertension by dehydrating agents with particular reference to the measurement of the blind spot area as a means of recording improvement. J. Neurol. Neurosurg. Psychiat. 39: 627-639.

Johnston I, Paterson A (1974) Benign intracranial hypertension. 1. Diagnosis and management. Brain 97: 289-300.

Keating JP, Feisin RD (1970) Raised intracranial pressure associated with probable Vitamin A deficiency in cystic fibrosis. Pediatr. 46: 41-46.

Lubow M, Kuhr L. Pseudotumor cerebri: Comments on practical management in Glaser JS (ed): Neuro-opthalmology, St . Louis, C.V. Mosby Co. 1977, pp 199-206.

Quincke H (1897) Uber Meningitis serosa and Verwandte zustande, Deutsche Ztschr Nervenh, 9: 140.

Roach ES, Sinal SH (1980) Increased intracranial pressure following treatment of cystic fibrosis. Pediatrics 66: 622-623.

Rose A, Matson DD (1967) Benign intracranial hypertension in children. Pediatrics 39: 227-237.

Rush J (1980) Pseudotumor cerebri. Clinical profile and visual outcome in 63 patients. Mayo Clin. Proc. 55: 541-546.

Sondheimer FS, Grossman H, Winchester P (1970) Suture distasis following rapid weight gain. Arch. Neurol. 23: 314-318.

Tibbles JAR, Vallet HL, Brown B St Je et al. (1972) Pseudotumor cerebri and deprivation dwarfism. Dev. Med. Child Neurol. 14: 322-331.

Weisberg LA (1975) Benign intracranial hypertension. Medicine 54: 197-207. 